\title{
Selective hippocampal cell damage and mossy fiber sprouting induced by chronic intracerebral injections of 2-deoxy-D-glucose
}

\author{
Evgeniia Samokhina, Anton Malkov, Alexander Samokhin and Irina Popova \\ Institute of Theoretical and Experimental Biophysics, Russian Academy of Sciences, Pushchino, Russia
}

\begin{abstract}
A reduction in glucose consumption has been shown in both patients with acquired epilepsy and in animal epilepsy models. However, the question remains whether the disturbance of glucose metabolism is the driving force of epileptogenesis. We have recently reported that a chronic partial inhibition of brain glycolysis by the non-metabolizable glucose analogue 2-deoxy-D-glucose (2-DG) triggers epileptogenesis in initially healthy rats. In this study, we further investigated whether chronic 2-DG treatment caused a cellular loss in the dorsal hippocampus and mossy fiber sprouting in the dentate gyrus. We found that prolonged (four weeks) treatment with 2-DG induced a neuronal loss in the CA1 field and the dentate hilus. We also found mossy fibers reorganization in the 2-DG group. In addition, we showed that pentylenetetrazole-induced convulsions were considerably strengthened and prolonged in 2-DG-treated rats. Our results demonstrate that the chronically impaired brain glucose metabolism likely leads to a structural remodeling resembling epileptogenesis and has a proconvulsive effect.
\end{abstract}

Key words: 2-DG - Epilepsy - Hippocampus - Pentylenetetrazole - Epileptogenesis

Abbreviations: 2-DG, 2-deoxy-D-glucose; PET, positron emission tomography; PTZ, pentyleneterazole; TLE, temporal lobe epilepsy.

\section{Introduction}

Epilepsy is a neurological disease characterized by spontaneous recurrent seizures (Fisher et al. 2014). Temporal lobe epilepsy (TLE) is the most common type of a drug-resistant partial epilepsy with seizures originating from or involving mesial temporal structures. Reduced glucose utilization during interictal periods detected by fluorine-18-tagged fluorodeoxyglucose (18 F-FDG) positron emission tomography (PET) technique is a generally recognized characteristic of the patients suffering from TLE (Pan et al. 2008; Cendes et al. 2016). Interestingly, genetically determined disruption of energy supply (mitochondrial disorders, creatine deficits and glucose transport deficiency) is known to be associated with different types of epileptic seizures (Reid et al. 2014). It

Correspondence to: Evgeniia Samokhina, Laboratory of Systemic Organization of Neurons, Institute of Theoretical and Experimental Biophysics of Russian Academy of Sciences, 3 Institutskaya Str., Pushchino, Moscow Region, 142290, Russia

E-mail: evgeniia.sam@iteb.ru has been proposed that the energy metabolism dysfunction (Kudin et al. 2009; Jupp et al. 2012; Lee et al. 2012; Reid et al. 2014), associated with oxidative stress (Azam et al. 2012; Martinc et al. 2012) and neuroinflammation (Wilcox and Vezzani 2014) is a trigger and driving force for acquired epileptogenesis (Zilberter and Zilberter 2017; Patel 2018).

The metabolic rate of glucose consumption increases during a seizure due to intensive neuronal firing (Wasterlain et al. 1993). During the postictal phase, however, glucose utilization is deficient (Guo et al. 2009; Jupp et al. 2012; Lee et al. 2012; Zhang et al. 2015). Moreover, the predictive value for the epilepsy outcome defined by the PET-identified glucose metabolism failure at an early phase of epileptogenesis has been demonstrated in different epilepsy models (Shultz et al. 2013; Bascuñana et al. 2016). Surprisingly, despite glucose hypometabolism is a generally recognised feature of epileptogenesis (Goffin et al. 2008; Pittau et al. 2014; Patel 2018), in the last decade an antiepileptic approach by glycolysis suppression has been suggested by a number of authors (Garriga-Canut et al. 2006; Stafstrom et al. 2009; Koenig et al. 2019; Rho et al. 2019), although a short time 
2-DG treatment revealed opposing effects (Stafstrom et al. 2008; Gasior et al. 2010; Rho et al. 2019), that is in agreement with ex vivo results demonstrating strong $2-\mathrm{DG}$ effects on multiple neuronal parameters underlying excitability, such as resting potential, synaptic transmission, energy metabolism and Cl- homeostasis (Zhao et al. 1997; Samokhina et al. 2017; Nedergaard and Andreasen 2018).

A rationale for such an approach was its apparent similarity to the low-carb, high-fat ketogenic diet (KD), which also leads to the suppression of glycolysis (Rho et al. 2019). However, in $\mathrm{KD}$, glucose is partially substituted by ketone bodies as a mitochondrial fuel (Bentourkia et al. 2009, Prince et al. 2013). Thus, the radical difference of KD and direct glycolysis inhibition is the absence of cellular energy deficiency in KD.

We have shown recently in vivo that chronic partial inhibition of brain glycolysis by 2 -DG significantly diminished the energy reserve store of glycogen and adenosine phosphates and induced epileptiform activity in initially healthy rats (Samokhina et al. 2017). Furthermore, a bidirectional positive feedback connecting seizures and energy failure has been shown in vitro (Malkov et al. 2018).

In this study, using 2-DG as a specific inhibitor of glycolysis (Chandramouli and Carter 1977), we investigated whether chronically reduced cellular glucose supply caused a neuronal loss and synaptic reorganization in the hippocampus. In addition, we treated rats with pentylenetetrazole (PTZ) to determine whether a chronic decrease in glycolysis could have a proconvulsive effect.

\section{Materials and Methods}

Animals

Experiments were performed on young adult male Wistar rats (250-300 g) obtained from the Experimental Animal
Center of the Institute of Theoretical and Experimental Biophysics (Pushchino, Russia). Animals were housed in pairs with food and water ad libitum. Animal handling and housing protocols were in accordance with the European Union guidelines (Directive 1986/609/EEC) for the use of experimental animals in scientific research. The protocol was approved by the Committee on the Bioethics of Animal Experiments of the Institute of Theoretical and Experimental Biophysics of the Russian Academy of Sciences.

All rats randomly allocated to three groups: 2-DG $(n=13)$, Saline $(n=15)$, and Control $(n=4)$. Rats in the 2 -DG group received $2.5 \mu \mathrm{l} 2$-DG $(20 \mathrm{mM})$ dissolved in sterile normal saline. Administration was daily for 28 days (Fig. 1A). Rats in the Saline group received equivalent volumes of $0.9 \%$ saline. Naive rats from the Control group were deprived of any neurosurgical and traumatic manipulations.

\section{Surgery}

Experimental animals underwent a neurosurgical operation (Fig. 1A) under general anesthesia (intramuscular injection): mixture (half-and-half Tiletamine hydrochloride and Zolazepam hydrochloride $(18 \mathrm{mg} / \mathrm{kg}$ ) and Xylazine hydrochloride $(12 \mathrm{mg} / \mathrm{kg})$. The body temperature was maintained by a heating pad and the cardiopulmonary parameters were monitored during the surgery by an Oxy9Vet Plus pulse oximeter (Bionet, South Korea). Rats were placed in a stereotaxic apparatus and a guide cannula (stainless steel, 21 gauge) was implanted above the left lateral brain ventricle ( $\mathrm{AP}=-0.8 ; \mathrm{L}=1.5 ; \mathrm{H}=3.0)$; according to the rat brain atlas (Paxinos and Watson 2007).

\section{Drugs and drug administration}

Drug administrations to animals were started after the post-surgical recovery period (Fig. 1A). 2-DG (purity 98\%; Sigma-Aldrich, Saint Louis, Missouri) was dissolved in saline
A

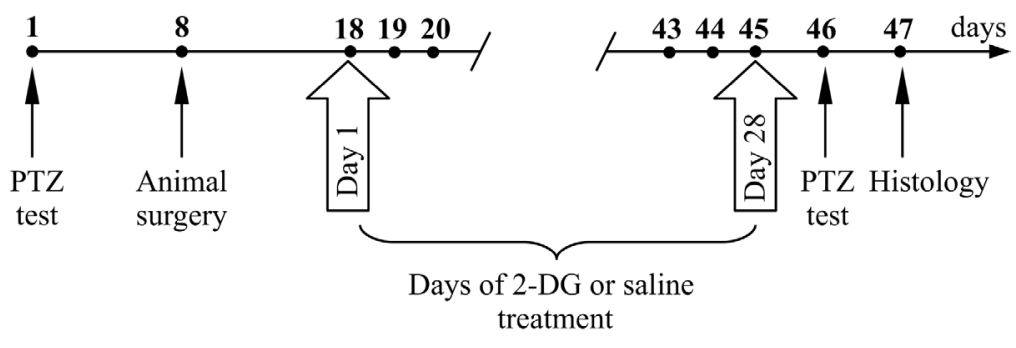

B

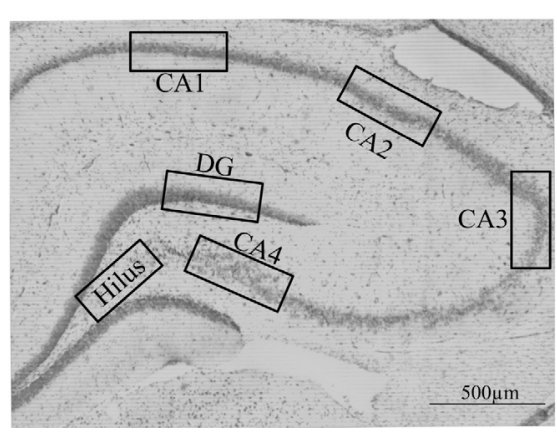

Figure 1. Experimental design. A. Timeline diagram showing the experimental protocol, including pentylenetetrazole (PTZ) seizure test, animal surgery, i.c.v. injections of 2-DG/saline and histological staining. B. Nissl-stained coronal slice of the dorsal hippocampus. Squares indicate the counting frames in the hippocampal fields (CA1, CA2, CA3, CA4), hilus and dentate gyrus (DG). 
solution (20 mM). 2-DG (2-DG group) or $0.9 \%$ saline solution (Saline group) injected intracerebroventricularly (i.c.v.) through the guide cannula to awaken rats using a Hamilton microsyringe type $75 \mathrm{~N}$ at an infusion rate of $1 \mu \mathrm{l} / \mathrm{min}$. For brain adaptation, the injection was usually started $1 \mathrm{~min}$ after the microsyringe insertion. The injection needle was slowly pulled out of the brain 2 min after the drug administration to avoid outflow. All substances were injected in equal volumes $(2.5 \mu \mathrm{l})$. The dose of $2-\mathrm{DG}$ was chosen based on our preliminary experiments (Samokhina et al. 2017).

\section{PTZ seizure test}

Rats from 2-DG and Saline groups were twice injected with pentylenetetrazole (PTZ). The proconvulsive agent PTZ was shown to stimulate seizure activity by blocking $\gamma$-aminobutyric acid (GABA)-mediated transmission (Ramzan and Levy 1985). Sub-threshold injections of PTZ ( $35 \mathrm{mg} / \mathrm{kg}$, intraperitoneal (i.p.)) were made: one week before surgery to estimate the pre-treatment seizure susceptibility and on the next day after the end of 2-DG or saline treatment to determine whether a chronic 2-DG treatment enhances susceptibility to PTZ (Fig. 1A). Following each PTZ injection, animals were placed into an acrylic glass transparent box, and behavioral seizures were monitored using a Sony Alpha5000 camera (Japan) for a period of $30 \mathrm{~min}$.

Because the chosen PTZ doze did not induce tonic-clonic convulsions in sufficient number of experimental rodents, two PTZ trails were conducted. In the first PTZ trail, seizure severity was estimated; in the second PTZ trail, latency time and duration of tonic-clonic convulsions were assessed. All results were analyzed in a blind manner.

\section{Histology}

On the next day after the final PTZ test, all animals were deeply anesthetized with an overdose of pentobarbital $(80 \mathrm{mg} / \mathrm{kg}$, i.p. $)$, perfused intracardially with $0.37 \%$ sodium sulfide in Timm buffer $\left(0.12 \mathrm{M} \mathrm{NaH}_{2} \mathrm{PO}_{4} \cdot 2 \mathrm{H}_{2} \mathrm{O}\right.$, $\left.0.10 \mathrm{M} \mathrm{NaOH}, 0.18 \mathrm{mM} \mathrm{CaCl}_{2}, \mathrm{pH} 7.4\right)$ for $20 \mathrm{~min}(15 \mathrm{ml} /$ min) followed by $4 \%$ paraformaldehyde solution for $20 \mathrm{~min}(15 \mathrm{ml} / \mathrm{min})$. Brains were removed from the skull and postfixed overnight in $4 \%$ paraformaldehyde at $4^{\circ} \mathrm{C}$. After cryoprotection in a gradient of sucrose (10\% and $20 \%$ sucrose in buffer at $4^{\circ} \mathrm{C}$ for $24 \mathrm{~h}$ each), brains were rapidly frozen in the vapor phase of liquid nitrogen. Coronal sections $(20 \mu \mathrm{m})$ were cut from the brain block with a Thermo Shandon Cryotome E (Thermo Scientific, USA) at $-19^{\circ} \mathrm{C}$ and collected onto poly-L-lysine-coated slides for subsequent Nissl and Timm staining. Nissl staining was used to verify cannula placements and to detect the safety of the left lateral ventricle and neuronal damage to the dorsal hippocampus (anterior posterior level between -3.5 and -4.5 according to the Paxinos and Watson (2007). Slide-mounted tissue sections were dried at room temperature overnight, submerged in bi-distilled water with acetate buffer for $5 \mathrm{~min}$, and stained in fresh $0.1 \%$ cresyl violet for 5-8 min until the desired depth of staining was achieved. To visualize supragranular mossy fiber sprouting in the dentate gyrus, Timm staining of 8-10 slices from each animal (2 slides) was performed at the levels corresponding to AP $=3.5-4.5$ as in (Paxinos and Watson 2007). Slide-mounted slices were developed using the following solution: $60 \mathrm{ml}$ of $50 \%$ gum arabic, $7.3 \mathrm{ml}$ of citrate buffer ( $2.55 \mathrm{~g}$ sodium citrate, $2.35 \mathrm{~g}$ citric acid), $30 \mathrm{ml}$ of $5.9 \%$ hydroquinone, and $0.5 \mathrm{ml}$ of $17 \%$ silver nitrate. The physical development was performed in the dark at $26^{\circ} \mathrm{C}$ for 60-90 min. Washing the slides in tap water for 10-15 min terminated the development of stain. The stained slides were dehydrated in graded ethanols, cleared in xylene, and coverslipped with Eukitt (Fluka, Germany) mounting medium.

Bright field images were acquired on a Nikon E200 microscope (Japan) with a Sony Alpha5000 camera (Japan). All tissue sections were photographed under identical conditions. Counts were performed on at least 5 sections. Cell quantifications were carried out manually using the "Cell counter" plugin of the ImageJ software (1.50i, USA) by an investigator who was blind to experimental group.

\section{Statistical analysis}

The results are presented as the mean \pm standard deviation. The $n$ values represent the number of animals used in the experiments. All statistical tests were performed using GraphPad Prism version 7 (GraphPad Software, Inc., USA). The D'Agostino-Pearson normality test (D'Agostino 1986) was applied in order to assess how far from Gaussian the data are. All data set fails the normality test as the $\mathrm{p}$ value is $\leq 0.05$, meaning that it deviates significantly from the normal distribution. Then, nonparametric statistical tests were executed. Statistical analysis of cell density in different areas of the hippocampus from the control and drug-treated groups was performed in two steps: statistically significant results by the Kruskal-Wallis test were analysed by the Dunn's post-hoc test. Changes in the severity of PTZ-induced seizures were tested by the Wilcoxon signed rank test for paired comparisons and Mann-Whitney $U$ test for independent data. $p \leq 0.05$ was considered statistically significant.

\section{Results}

\section{PTZ sensitivity test}

Seizures induced by PTZ were scored as follows: stage 0 , no response; stage 1 , ear and facial twitching; stage 2, convulsive waves moving axially through the body; stage 3 , 
Table 1. The effects of chronic treatment with 2-deoxy-D-glucose (2-DG) and saline on susceptibility to seizure induced by PTZ (35 mg/kg, i.p.).

\begin{tabular}{|c|c|c|c|c|c|}
\hline \multirow{2}{*}{ Group } & \multicolumn{2}{|c|}{ Seizure stage } & \multicolumn{2}{|c|}{ Latency time (s) to } & \multirow{2}{*}{$\begin{array}{l}\text { Duration tonic } \\
\text { convulsions }(\mathrm{s})\end{array}$} \\
\hline & before treatment & after treatment & 2nd seizure stage & 4th seizure stage & \\
\hline Saline & $\begin{array}{l}1.3 \pm 1.2 \\
(n=15)\end{array}$ & $\begin{array}{l}2.6 \pm 1.7 \\
(n=15) \\
\end{array}$ & $\begin{array}{c}62.00 \pm 32.47 \\
(n=15)\end{array}$ & $\begin{array}{c}455 \pm 155.37 \\
(n=4)\end{array}$ & $\begin{array}{c}35.25 \pm 12.70 \\
(n=4)\end{array}$ \\
\hline $2-\mathrm{DG}$ & $\begin{array}{l}0.8 \pm 1.3 \\
(n=13)\end{array}$ & $\begin{array}{c}3.6 \pm 0.8^{\S} \\
(n=13)\end{array}$ & $\begin{array}{c}65.83 \pm 30.00 \\
(n=13)\end{array}$ & $\begin{array}{c}422.33 \pm 189.39 \\
(n=6)\end{array}$ & $\begin{array}{c}76.5 \pm 11.28^{*} \\
(n=6)\end{array}$ \\
\hline
\end{tabular}

Chronic 2-DG treatment induced a significant difference in seizure stages $v$ s. seizure stages before treatment $\left({ }^{\S} p=0.03\right.$, Wilcoxon signed paired test) as well as the duration of stage $4 / 5$ seizures as compared to saline-treated rats $\left({ }^{*} p=0.03\right.$, Mann-Whitney $\mathrm{U}$ test). PTZ, pentyleneterazole; $n$, the total number of animals.

myoclonic jerks and rearing; stage 4, clonic convulsions with the animal falling on its side; stage 5 , repeated severe tonic-clonic convulsions or lethal convulsions. As shown in Table 1, there was a significant difference between the seizure stage after chronic 2-DG treatment and the initial seizure stage (before treatment): $0.8 \pm 1.3$ before $2-\mathrm{DG}$ treatment $(n=13)$ and $3.6 \pm 0.8$ after 4 weeks of daily 2 -DG injections $(n=13),(p=0.03$, Wilcoxon signed paired test). In the Saline group, convulsions did not significantly intensify during four experimental weeks (Table 1). Also we observed a significant increase in the duration of clonic convulsions in the 2-DG group in comparison with the saline-treated group: from $35.25 \pm 12.70$ (s) in the Saline group $(n=4)$ to $76.5 \pm 11.28$ (s) in the $2-\mathrm{DG}$ group $(n=$ $6),(p=0.03$, Mann-Whitney U test). However, our analysis did not reveal statistically significant differences in the latency time for behavioural seizures between these two groups of animals.
A

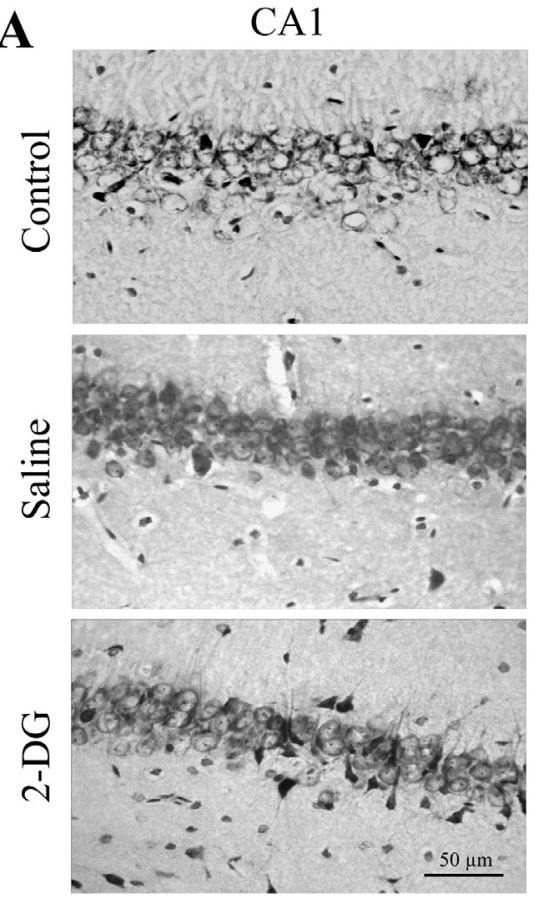

Hilus
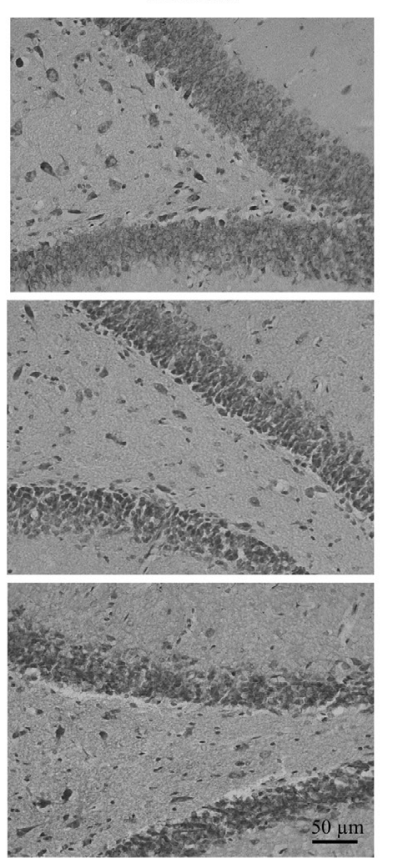

B

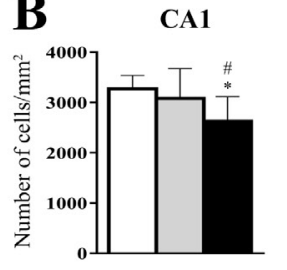

D

CA3

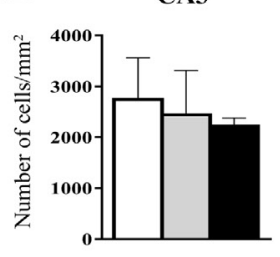

F

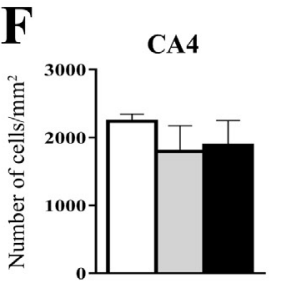

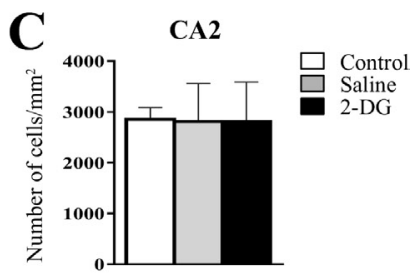

$\mathbf{E}$
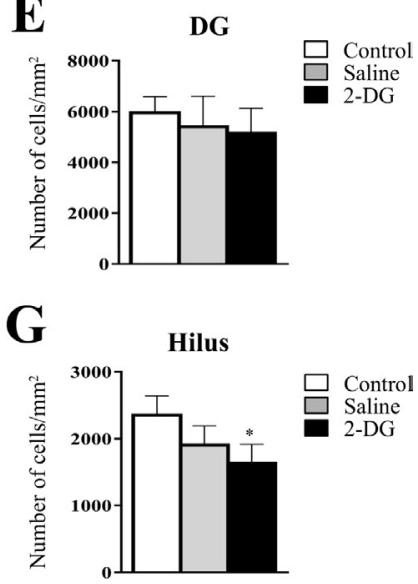

Figure 2. Nissl-stained sections and quantitative analysis of cell density in the dorsal hippocampus. 28 days following 2-DG treatment, neuronal loss was found in the hilus (A, G) $\left(^{*} p=0.01 v s\right.$. Control) and field CA1 (A, B) of the 2-DG group $\left({ }^{*} p=0.03\right.$ and ${ }^{\#} p=0.04 v s$. Control and Saline groups, respectively). The cell densities in other hippocampal fields (CA2, CA3, CA4) and dentate gyrus (D, G) in the 2-DG group did not differ from those in the Control or Saline groups (C, D, E, F). Each bar represents the mean \pm SD. No statistically significant difference in the average cell density was revealed in saline-treated animals vs. control animals in all analyzed hippocampal fields. Kruskal-Wallis test followed by Dunn's post-hoc test was performed. 

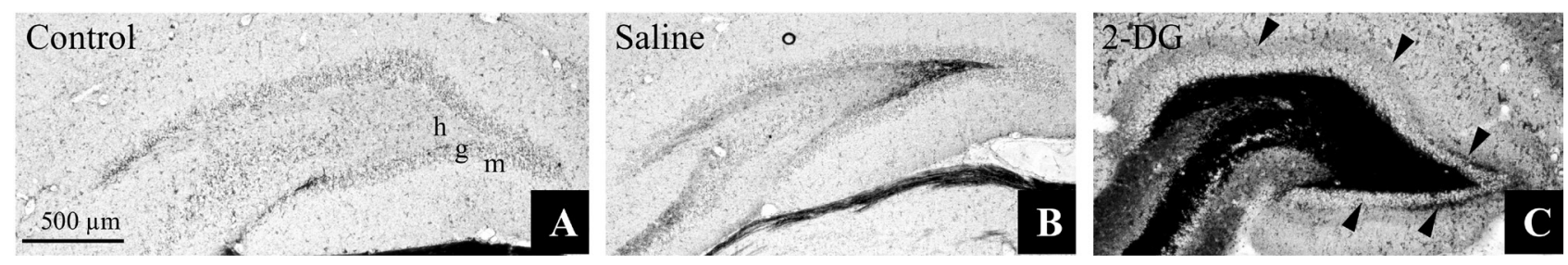

Figure 3. Sections of the dorsal hippocampus stained by the Timm method for identification of mossy fiber sprouting in the dentate gyrus. These sections demonstrate Timm-negative stain from the Control animal group without any injection (A); from the Saline group (B) and Timm-positive stain (arrowheads) in the dentate inner molecular layer of the 2-DG group (C). h, hilus; g, granule cell layer; $\mathrm{m}$, molecular layer.

\section{Neuronal loss in hippocampus}

Assessment of neuronal density was performed on the Nissl-stained sections. Neuronal quantification was carried out in the dentate gyrus, hippocampal pyramidal cell layers: fields CA1, CA2, CA3, CA4 (counting frame 7,225 $\mu \mathrm{m}^{2}$ ) and dentate hilus (counting frame 14,450 $\mathrm{m}^{2}$ ) (Fig. 1B).

Four weeks after the 2-DG treatment, light microscopy showed that the degradation of the pyramidal layer in CA1 reached of $20 \%$ of Control group $(p=0.03)$ and $15 \%$ of Saline group $(p=0.04)$ : cell density in the Control $(n=4)$ and Saline group $(n=15)$ were $3271 \pm 264$ and $3082 \pm 594$, respectively, as compared to $2631 \pm 482$ in the 2-DG ( $n=$ 13) (Fig. 2A, B). In the 2-DG group, the cell density in the hilus of the dentate gyrus was also considerably reduced: the neuron loss was 31\% for the 2-DG group relative to the Control (Fig. 2A, G). The cell density was $2352 \pm 285$ in the Control $(n=4)$ as compared to $1636 \pm 279$ in the 2-DG group $(n=7, p=0.01)$. Importantly, the cell densities in CA2, CA3, CA4, and dentate gyrus in the 2-DG group did not differ from those in the Control or Saline groups (Fig. 2 C-F). No statistically significant differences in the average densities of neurons were shown in saline-treated animals $v s$. naive animals in all analyzed hippocampal fields.

Importantly, neuronal densities in CA2, CA3, CA4 and dentate gyrus in the 2-DG group did not differ from those in the Saline group (Fig. 2C-F). No statistically significant differences in the average densities of neurons were shown in saline-treated animals vs. naive animals in all analyzed hippocampal fields except hilus.

\section{Detection of mossy fiber sprouting}

Timm staining was performed to measure the mossy fiber sprouting in hippocampus. The degree of mossy fiber sprouting was rated using semi-quantitative analysis (Cavazos et al. 1991) as follows: (1) sparse Timm granules in the supragranular zone; (2) more numerous granules in continuous distribution; (3) prominent granules and patches; (4) a dense laminar band in the supragranular layer; and
(5) a dense laminar band extending to the inner molecular layer. Representative images of Timm staining are shown for three groups: Control ( $n=4$; Fig. 3A), Saline ( $n=9$; Fig. 3B), and 2-DG $(n=12$; Fig. $3 \mathrm{C})$. The results indicated that aberrant mossy fiber sprouting from the dentate gyrus toward the inner molecular layer was significantly increased in the 2-DG group (2.16 $\pm 1.11, p=0.02)$ (Fig. 4$)$ compared to the Saline group (1.11 \pm 0.92$)$ (Fig. 4). In naive control rats $(n=$ 4), there was a subtle black Timm staining in the granule cell layer and absence of aberrant mossy fiber sprouting into the inner molecular layer $(0.75 \pm 0.5)$. No statistically significant differences were found in Saline vs. Control group.

\section{Discussion}

A novel finding of this study indicates that chronically reduced cerebral glucose utilization via i.c.v. 2-DG injection leads to the hippocampal neuron loss and synaptic reorganization. Specifically, we demonstrate that chronic 2-DG treatment (daily for 28 days) induces a significant reduction of neuronal density in both the hippocampal

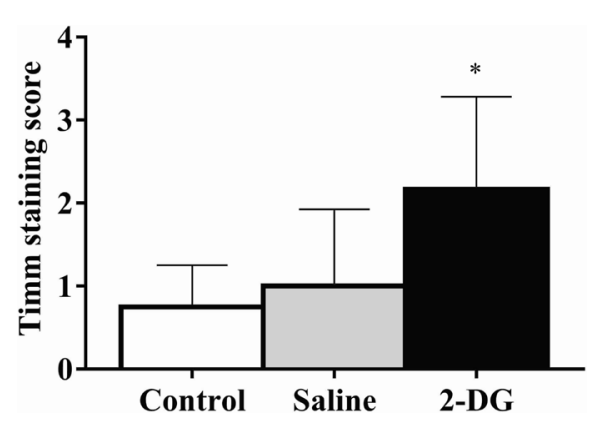

Figure 4. Timm staining scores of rats after 4 weeks of 2-DG/saline treatment. Horizontal bars represent the group means. Each column corresponds to the mean group score $\pm \mathrm{SD}$. The score difference between 2-DG $(n=12)$ and Saline $(n=9)$ groups was statistically significant $\left({ }^{*} p=0.02\right)$. Kruskal-Wallis test followed by Dunn's post-hoc test was performed. 
CA1 field and the hilus of dentate gyrus. We also show that the 2-DG treatment initiates sprouting of mossy fibers in the inner third part of dentate gyrus molecular layer. Thus, the chronic energy deficiency state created by 2-DG leads to structural and irreversible changes in nervous tissue that are specific for epileptogenesis.

Hippocampal sclerosis, a selective neuronal loss in the CA1/CA3 region of hippocampus and hilus, is a characteristic feature of TLE patients and animal epilepsy models (Berkovic et al. 1991; Curia et al. 2008; Lévesque and Avoli 2013). Interestingly, in our work, neuronal loss in CA1 and dentate hilus was more dramatic, whereas a reduction of cell density in CA3 was significantly less pronounced. Whether CA3 area is more resistant to glucose metabolism breakdown is unknown. One possibility is that the CA1 region exhibits fewer capillaries than the neighbouring CA3 region (Cavaglia et al. 2001), thus higher stability of CA3 area may be caused by higher vascularization. Previously, it was suggested that CA3 receives two major vascular afferents (dorsal arteries) while CA1 is vascularized by only one arterial branch (ventral artery), that may be the underlying anatomical source of capillary density difference (Marinkovic et al. 1992). Given that hippocampal neurons demonstrate different degree of sensitivity to 2-DG treatment, it will be interesting to explore this phenomenon in future studies.

Most notably, we have shown for the first time that 2-DG treatment induces the development of mossy fiber sprouting in the dentate gyrus of hippocampus. In contrast, morphological alterations were negligible in saline-treated animals. Additionally, in the 2-DG-treated group, we showed a robust decline of threshold for inducing seizures in the PTZ test. This is consistent with literature data showing that acute 2-DG treatment does not protect against pentylenetetrazole-, kainic acid- or maximal electroshock-induced seizures (Lian et al. 2007; Stafstrom et al. 2009; Gasior et al. 2010). While 2-DG induced synaptic reorganization and atrophy in the hippocampal regions, chronic implantation of the intraventricular cannula as well as daily i.c.v. injections did not induced neuronal density decline, mossy fiber sprouting or a pro-epileptic effect in the PTZ test. Therefore, the invasive approach we used in experiments did not result itself to posttraumatic epileptogenesis.

An important experimental aspect is the mode of 2-DG administration. In our work, the injection of 2-DG was i.c.v., directly inhibiting glycolysis in brain cells and causing chronic energy deficiency in the brain. In contrast, studies showing the protective role of 2-DG treatment (Shao et al. 2018; Rho et al. 2019) used i.p. drug administration, causing a concurrent and significant rise of both blood glucose level and cerebral blood flow, as has been reported for both animals (Combs et al. 1986; Breier et al. 1993; Horinaka et al. 1997; Koenig et al. 2019; Leiter et al. 2019) and humans (Landau et al. 1958; Elman et al. 1999). Importantly, the brain 2-DG concentration was not estimated in these studies but seems to be insufficient for providing the direct effect on brain cell energy metabolism (Samokhina et al. 2017). Therefore, the 2-DG brain effects in the studies using i.p. drug administration were most likely indirect, and referencing in these studies a 2-DG brain glycolysis inhibition is obviously inadequate. Our results show directly the contribution of brain glucose hypometabolism to the development of pathology and confirm the view on acquired epilepsy as a metabolic disease (Zilberter and Zilberter 2017; Patel 2018).

Here we show that chronic 2-DG-induced inhibition of glycolysis results in changes in neuronal density and synaptic reorganization in the hippocampus. We also demonstrate that the chronic 2-DG treatment has a pro-epileptic effect. These results show that the chronic decrease in brain energy metabolism and, in particular, reduction in glucose utilization leads to irreversible structural rearrangements in the nervous tissue, that is important for both the understanding general mechanisms of brain pathogenesis and the development of therapeutic approaches aimed at eliminating the cause, rather than the symptoms, of neurological diseases.

Acknowledgements. The investigation of chronic 2-DG and saline treatment was supported by the Russian Foundation for Basic Research (grant numbers 18-315-00225). The histological assessment of control animal group was supported by RSF (project No. 17-75-20245). All authors were involved in the design of the study, data collection and analysis, decisions to publish, and preparation of the manuscript. The authors are grateful to Dr. Yuri Zilberter for comments and help in preparing the manuscript.

Conflicts of interest. There are no actual or potential conflicts of interest.

\section{References}

Azam F, Prasad MV, Thangavel N (2012): Targeting oxidative stress component in the therapeutics of epilepsy. Curr. Top Med. Chem. 12, 994-1007 https://doi.org/10.2174/156802612800229224

Bascuñana P, Javela J, Delgado M, Fernández de la Rosa R, Shiha AA, García-García L, Pozo MÁ (2016): [18F]FDG PET Neuroimaging predicts pentylenetetrazole (PTZ) kindling outcome in rats. Mol. Imaging Biol. 18, 733-740 https://doi.org/10.1007/s11307-016-0950-0

Bentourkia M, Tremblay S, Pifferi F, Rousseau J, Lecomte R, Cunnane S (2009): PET study of 11C-acetoacetate kinetics in rat brain during dietary treatments affecting ketosis. Am. J. Physiol. Endocrinol. Metab. 296, 796-801 https://doi.org/10.1152/ajpendo.90644.2008

Berkovic SF, Andermann F, Olivier A, Ethier R, Melanson D, Robitaille Y, Kuzniecky R, Peters T, Feindel W (1991): Hippocampal sclerosis in temporal lobe epilepsy demonstrated by magnetic resonance imaging. Ann. Neurol. 29, 175-182 https://doi.org/10.1002/ana.410290210 
Breier A, Crane AM, Kennedy C, Sokoloff L (1993): The effects of pharmacologic doses of 2-deoxy-D-glucose on local cerebral blood flow in the awake, unrestrained rat. Brain Res. 618, $277-282$ https://doi.org/10.1016/0006-8993(93)91276-X

Cavaglia M, Dombrowski SM, Drazba J, Vasanji A, Bokesch PM, Janigro D (2001): Regional variation in brain capillary density and vascular response to ischemia. Brain Res. 910, 81-93 https://doi.org/10.1016/S0006-8993(01)02637-3

Cavazos JE, Golarai G, Sutula TP (1991): Mossy fiber synaptic reorganization induced by kindling: time course of development, progression, and permanence. J. Neurosci. 11, 27952803

https://doi.org/10.1523/JNEUROSCI.11-09-02795.1991

Cendes F, Theodore WH, Brinkmann BH, Sulc V, Cascino GD (2016): Neuroimaging of epilepsy. Handbook of clinical neurology. 136, 985-1014 https://doi.org/10.1016/B978-0-444-53486-6.00051-X

Chandramouli V, Carter JR Jr (1977): Metabolic effects of 2-deoxy- D -glucose in isolated fatcells, Biochim. Biophys. Acta 496, 278-291 https://doi.org/10.1016/0304-4165(77)90310-5

Combs DJ, Reuland DS, Martin DB, Zelenock GB, D‘Alecy LG (1986): Glycolytic inhibition by 2-deoxyglucose reduces hyperglycemiaassociated mortality and morbidity in the ischemic rat. Stroke 17, 989-994 https://doi.org/10.1161/01.STR.17.5.989

Curia G, Longo D, Biagini G, Jones RS, Avoli M (2008): The pilocarpine model of temporal lobe epilepsy. J. Neurosci. Methods 172, 143-157 https://doi.org/10.1016/j.jneumeth.2008.04.019

D’Agostino RB (1986): Tests for normal distribution. In: Goodnessof-fit Techniques. (Eds. RB D’Agostino, MA Stephens), pp. 367-420, Marcel Dekker, New York. https://doi.org/10.1201/9780203753064-9

Elman I, Sokoloff L, Adler CM, Weisenfeld N, Breier A (1999): The effects of pharmacological doses of 2-deoxyglucose on cerebral blood flow in healthy volunteers. Brain Res. 815, 243-249. https://doi.org/10.1016/S0006-8993(98)01137-8

Fisher RS, Acevedo C, Arzimanoglou A, Bogacz A, Cross JH, Elger CE, Engel JJr, Forsgren L, French JA, Glynn M, et al. (2014): ILAE official report: a practical clinical definition of epilepsy. Epilepsia 55, 475-482 https://doi.org/10.1111/epi.12550

Garriga-Canut M, Schoenike B, Qazi R, Bergendahl K, Daley TJ, Pfender RM, Morrison JF, Ockuly J, Stafstrom C, Sutula T, Roopra A (2006): 2-Deoxy-D-glucose reduces epilepsy progression by NRSF-CtBP-dependent metabolic regulation of chromatin structure. Nat. Neurosci. 9, 1382-1387 https://doi.org/10.1038/nn1791

Gasior M, Yankura J, Hartman AL, French A, Rogawski MA (2010): Anticonvulsant and proconvulsant actions of 2-deoxy-Dglucose. Epilepsia 51, 1385-1394 https://doi.org/10.1111/j.1528-1167.2010.02593.x

Goffin K, Dedeurwaerdere S, Van Laere K, Van Paesschen W (2008): Neuronuclear assessment of patients with epilepsy. Semin. Nucl. Med. 38, 227-239 https://doi.org/10.1053/j.semnuclmed.2008.02.004
Guo Y, Gao F, Wang S, Ding Y, Zhang H, Wang J, Ding MP (2009): In vivo mapping of temporospatial changes in glucose utilization in rat brain during epileptogenesis: an 18F-fluorodeoxyglucosesmall animal positron emission tomography study. Neuroscience 162, 972-979 https://doi.org/10.1016/j.neuroscience.2009.05.041

Horinaka N, Artz N, Jehle J, Takahashi S, Kennedy C, Sokoloff L (1997): Examination of potential mechanisms in the enhancement of cerebral blood flow by hypoglycemia and pharmacological doses of deoxyglucose. J. Cereb. Blood Flow Metab. 17, 54-63 https://doi.org/10.1097/00004647-199701000-00008

Jupp B, Williams J, Binns D, Hicks RJ, Cardamone L, Jones N, Rees S, O'Brien TJ (2012): Hypometabolism precedes limbic atrophy and spon-taneous recurrent seizures in a rat model of TLE. Epilepsia 53, 1233-1244 https://doi.org/10.1111/j.1528-1167.2012.03525.x

Koenig JB, Cantu D, Low C, Sommer M, Noubary F, Croker D, Whalen M, Kong D, Dulla CG (2019): Glycolytic inhibitor 2-deoxyglucose prevents cortical hyperexcitability after traumatic brain injury. JCI Insight 30, 5-19 https://doi.org/10.1172/jci.insight.126506

Kudin AP, Zsurka G, Elger CE, Kunz WS (2009): Mitochondrial involvement in temporal lobe epilepsy. Exp. Neurol. 218, 326-332 https://doi.org/10.1016/j.expneurol.2009.02.014

Landau BR, Laszlo J, Stengle J, Burk D (1958): Certain metabolic and pharmacologic effects in cancer patients given infusions of 2-deoxy-D-glucose. J. Natl. Cancer Inst. 21, 485-494

Lee EM, Park GY, Im KC, Kim ST, Woo CW, Chung JH, Kim KS, Kim JS, Shon YM, Kim YI, Kang JK (2012): Changes in glucose metabolism and metabolites during the epileptogenic process in the lithium-pilocarpine model of epilepsy. Epilepsia 53, 860-869 https://doi.org/10.1111/j.1528-1167.2012.03432.x

Leiter I, Bascuñana P, Bengel FM, Bankstahl JP, Bankstahl M (2019): Attenuation of epileptogenesis by 2-deoxy-D-glucose is accompanied by increased cerebral glucose supply, microglial activation and reduced astrocytosis. Neurobiol. Dis. 130, 104510 https://doi.org/10.1016/j.nbd.2019.104510

Lévesque M, Avoli M (2013): The kainic acid model of temporal lobe epilepsy. Neurosci. Biobehav. Rev. 37, 2887-2899 https://doi.org/10.1016/j.neubiorev.2013.10.011

Lian XY, Khan FA, Stringer JL (2007): Fructose-1,6-bisphosphate has anticonvulsant activity in models of acute seizures in adult rats. J. Neurosci. 27, 12007-12011 https://doi.org/10.1523/JNEUROSCI.3163-07.2007

Malkov A, Ivanov AI, Buldakova S, Waseem T, Popova I, Zilberter M, Zilberter Y (2018): Seizure-induced reduction in glucose utilization promotes brain hypometabolism during epileptogenesis. Neurobiol. Dis. 116, 28-38 https://doi.org/10.1016/j.nbd.2018.04.016

Marinkovic S, Milisavljevic M, Puskas L (1992): Microvascular anatomy of the hippocampal formation. Surg. Neurol. 37, 339-349 https://doi.org/10.1016/0090-3019(92)90001-4

Martinc B, Grabnar I, Vovk T (2012): The role of reactive species in epileptogenesis and influence of antiepileptic drug therapy on oxidative stress. Curr. Neuropharmacol. 10, 328-343 https://doi.org/10.2174/157015912804499447 
Nedergaard S, Andreasen M (2018): Opposing effects of 2-DeoxyD-Glucose on interictal- and ictal-like activity when $\mathrm{K}+$ currents and GABAA receptors are blocked in rat hippocampus in vitro. J. Neurophysiol. 119, 1912-1923 https://doi.org/10.1152/jn.00732.2017

Pan JW, Williamson A, Cavus I, Hetherington HP, Zaveri H, Petroff OA, Spencer DD (2008): Neurometabolism in human epilepsy. Epilepsia 49, 31-41 https://doi.org/10.1111/j.1528-1167.2008.01508.x

Patel M (2018): A metabolic paradigm for epilepsy. Epilepsy Currents/American Epilepsy Society 18, 318-322 https://doi.org/10.5698/1535-7597.18.5.318

Paxinos G, Watson C (2007): The Rat Brain in Stereotaxic Coordinates. Ed. 6, Academic Press, San Diego

Pittau F, Grouiller F, Spinelli L, Seeck M, Michel CM, Vulliemoz $S$ (2014): The role of functional neuroimaging in pre-surgical epilepsy evaluation. Front. Neurol. 5, 31 https://doi.org/10.3389/fneur.2014.00031

Prince A, Zhang Y, Croniger C, Puchowicz M (2013): Oxidative metabolism: glucose versus ketones. Adv. Exp. Med. Biol. 789, 323-328 https://doi.org/10.1007/978-1-4614-7411-1_43

Ramzan IM, Levy G (1985): Kinetics of drug action in disease states. XIV. Effect of infusion rate on pentylenetetrazol concentrations in serum, brain and cerebrospinal fluid of rats at onset of convulsions. J. Pharmacol. Exp. Ther. 234, 624-628

Reid CA, Mullen S, Kim TH, Petrou S (2014): Epilepsy, energy deficiency and new therapeutic approaches including diet. Pharmacol. Ther. 144, 192-201 https://doi.org/10.1016/j.pharmthera.2014.06.001

Rho JM, Shao LR, Stafstrom CE (2019): 2-Deoxyglucose and betahydroxybutyrate: metabolic agents for seizure control. Front. Cell. Neurosci. 13, 172 https://doi.org/10.3389/fncel.2019.00172

Samokhina E, Popova I, Malkov A, Ivanov AI, Papadia D, Osypov A, Molchanov M, Paskevich S, Fisahn A, Zilberter M, Zilberter Y (2017): Chronic inhibition of brain glycolysis initiates epileptogenesis. J. Neurosci. Res. 95, 2195-2206 https://doi.org/10.1002/jnr.24019

Shao LR, Rho JM, Stafstrom CE (2018): Glycolytic inhibition: a novel approach toward controlling neuronal excitability and seizures. Epilepsia Open 3, 191-197 https://doi.org/10.1002/epi4.12251

Shultz SR, Cardamone L, Liu YR, Hogan RE, Maccotta L, Wright DK, Zheng P, Koe A, Gregoire MC, Williams JP, et al. (2013): Can structural or functional changes following traumatic brain injury in the rat predict epileptic outcome? Epilepsia 54, $1240-1250$

https://doi.org/10.1111/epi.12223

Stafstrom CE, Roopra A, Sutula TP (2008): Seizure suppression via glycolysis inhibition with 2-deoxy-D-glucose (2DG). Epilepsia 49, 97-100 https://doi.org/10.1111/j.1528-1167.2008.01848.x

Stafstrom CE, Ockuly JC, Murphree L, Valley MT, Roopra A, Sutula TP (2009): Anticonvulsant and antiepileptic actions of 2-deoxy-D-glucose in epilepsy models. Ann. Neurol. 65, 435-447

https://doi.org/10.1002/ana.21603

Wasterlain CG, Fujikawa DG, Penix L, Sankar R (1993): Pathophysiological mechanisms of brain damage from status epilepticus. Epilepsia 34, 37-53 https://doi.org/10.1111/j.1528-1157.1993.tb05905.x

Wilcox KS, Vezzani A (2014): Does brain inflammation mediate pathological outcomes in epilepsy? Adv. Exp. Med. Biol. 813, 169-183 https://doi.org/10.1007/978-94-017-8914-1_14

Zhang L, Guo Y, Hu H, Wang J, Liu Z, Gao F (2015): FDG-PET and NeuN-GFAP immunohistochemistry of hippocampus at different phases of the pilocarpine model of temporal lobe epilepsy. Int. J. Med. Sci. 12, 288-294

https://doi.org/10.7150/ijms.10527

Zhao YT, Tekkok S, Krnjevic K (1997): 2-Deoxy-D-glucoseinduced changes in membrane potential, input resistance, and excitatory postsynaptic potentials of CA1 hippocampal neurons. Can. J. Physiol. Pharmacol. 75, 368-374 https://doi.org/10.1139/y97-073

Zilberter Y, Zilberter M (2017): The vicious circle of hypometabolism in neurodegenerative diseases: ways and mechanisms of metabolic correction. J. Neurosci. Res. 95, 2217-2235 https://doi.org/10.1002/jnr.24064

Received: March 3, 2019

Final version accepted: October 29, 2019 